\title{
Particularities of preimmune reactivity in patients with pulmonary tuberculosis in combination with toxocariasis
}

\author{
Valentina Smesnoi ${ }^{1,2,3^{*}}$, Serghei Ghindă' ${ }^{1}$ Gheorghe Placintă ${ }^{2,3}$ \\ From The 9th Edition of the Scientific Days of the National Institute for Infectious Diseases Prof Dr Matei \\ Bals \\ Bucharest, Romania. 23-25 October 2013
}

\section{Background}

For many years it was considered that bronchial obstruction is caused by a bacterial or viral infection of the respiratory system and the pathogenesis is associated with the sensitization of a bronchial tree by pathogens or non-pathogenic entities which inhabit the respiratory passages. Symptomatic pulmonary disease characteristics are present in $65 \%$ of patients with visceral toxocariasis and catarrhal symptoms vary from mild to severe asthmatic.

\section{Methods}

The study included 66 patients, out of which 20 had pulmonary tuberculosis, 20 had pulmonary tuberculosis and Toxocara coinfection, and 26 Toxocara infection. The levels of cytokines IL-4, anti-Toxocara IgG and IgE were determined by ELISA. To assess the functional activity of neutrophils the test nitro-blue tetrazolium (NBT) was used and we modified the method proposed by Park.

\section{Results}

The study of cytokine profile (IL-4), Ig E levels, eosinophils and $\mathrm{T}$ lymphocytes proliferative activity in the reaction of blast transformation of lymphocytes demonstrated that for patients with tuberculosis and toxocariasis association the Th1/Th2 type immune response is characteristic. For patients with pulmonary tuberculosis the Th1 type immune response is characteristic, while for patients with toxocariasis is characteristic the Th2 type of immune response.

\section{Conclusion}

In patients with toxocariasis without respiratory pathology we identified Th2 type of immune response to treatment and its dynamics. This could explain a more favorable prognosis for a respiratory pathology associated with toxocariasis.

\section{Authors' details}

${ }^{1}$ Chiril Draganiuc Institute of Phthisiopneumology, Chişinău, Republic of Moldova. ${ }^{2}$ Department of Infectious Diseases, Faculty for Continuing Medical Education, Nicolae Testemițanu State Medical and Pharmacy University, Chişinău, Republic of Moldova. ${ }^{3}$ Toma Ciorbă Clinical Hospital for Infectious Diseases, Chişinău, Republic of Moldova.

Published: 16 December 2013

doi:10.1186/1471-2334-13-S1-P80

Cite this article as: Smesnoi et al:: Particularities of preimmune reactivity in patients with pulmonary tuberculosis in combination with toxocariasis. BMC Infectious Diseases 2013 13(Suppl 1):P80.

\footnotetext{
* Correspondence: valentina_smesnoi@yahoo.com

${ }^{1}$ Chiril Draganiuc Institute of Phthisiopneumology, Chişinău, Republic of Moldova

Full list of author information is available at the end of the article
} 